\title{
Spinal cord stimulation reduces cardiac pain through microglial deactivation in rats with chronic myocardial ischemia
}

\author{
JIAN WANG $^{1 *}$, XIAO-CHEN WU ${ }^{1 *}$, MING-MING ZHANG ${ }^{2 *}$, JIA-HAO REN $^{2}$, YI SUN $^{2}$, \\ JING-ZHEN LIU ${ }^{1}$, XI-QIANG WU ${ }^{1}$, SI-YI HE ${ }^{1}$, YUN-QING LI $^{2}$ and JIN-BAO ZHANG ${ }^{1}$ \\ ${ }^{1}$ Department of Cardiothoracic Surgery, General Hospital of Western Theater Command, \\ Chengdu, Sichuan 610083; ${ }^{2}$ Department of Anatomy and K.K. Leung Brain Research Centre, \\ Air Force Military Medical University, Xi'an, Shaanxi 710032, P.R. China
}

Received July 15, 2021; Accepted September 7, 2021

DOI: $10.3892 / \mathrm{mmr} .2021 .12475$

\begin{abstract}
Angina pectoris is cardiac pain that is a common clinical symptom often resulting from myocardial ischemia. Spinal cord stimulation (SCS) is effective in treating refractory angina pectoris, but its underlying mechanisms have not been fully elucidated. The spinal dorsal horn is the first region of the central nervous system that receives nociceptive information; it is also the target of SCS. In the spinal cord, glial (astrocytes and microglia) activation is involved in the initiation and persistence of chronic pain. Thus, the present study investigated the possible cardiac pain-relieving effects of SCS on spinal dorsal horn glia in chronic myocardial ischemia (CMI). CMI was established by left anterior descending artery ligation surgery, which induced significant spontaneous/ongoing cardiac pain behaviors, as measured using the open field test in rats. SCS effectively improved such behaviors as shown by open field and conditioned place preference tests in CMI model rats. SCS suppressed CMI-induced spinal dorsal horn microglial activation, with downregulation of ionized calcium-binding adaptor protein-1 expression. Moreover, SCS inhibited CMI-induced spinal expression of phosphorylated-p38 MAPK, which was specifically colocalized with the spinal dorsal horn microglia rather than astrocytes and neurons. Furthermore, SCS could
\end{abstract}

Correspondence to: Dr Yun-Qing Li, Department of Anatomy and K.K. Leung Brain Research Centre, Air Force Military Medical University, 169 Changle West Road, Xincheng, Xi'an, Shaanxi 710032, P.R. China

E-mail: deptanat@fmmu.edu.cn

Dr Jin-Bao Zhang, Department of Cardiothoracic Surgery, General Hospital of Western Theater Command, 270 Rongdu Avenue, Jinniu, Chengdu, Sichuan 610083, P.R. China

E-mail: zjbwyw@sina.com

${ }^{*}$ Contributed equally

Key words: cardiac pain, chronic myocardial ischemia, spinal cord stimulation, microglia depress spinal neuroinflammation by suppressing CMI-induced IL- $1 \beta$ and TNF- $\alpha$ release. Intrathecal administration of minocycline, a microglial inhibitor, alleviated the cardiac pain behaviors in CMI model rats. In addition, the injection of fractalkine (microglia-activating factor) partially reversed the SCS-produced analgesic effects on CMI-induced cardiac pain. These results indicated that the therapeutic mechanism of SCS on CMI may occur partially through the inhibition of spinal microglial p38 MAPK pathway activation. The present study identified a novel mechanism underlying the SCS-produced analgesic effects on chronic cardiac pain.

\section{Introduction}

Patients suffering from moderate to severe angina pectoris, which often results from chronic myocardial ischemia (CMI) despite optimal medical therapy, and judged unsuited to both percutaneous coronary intervention and coronary artery bypass graft surgery, are a challenging issue for cardiologists (1). Such pain severely limits daily activities and impairs the quality of life among these patients (2). Owing to the current inadequate understanding of the underlying mechanisms, the management of chronic cardiac pain remains difficult.

It has been shown that cardiac visceral nociceptive information is preliminarily transmitted to the spinal dorsal horn of thoracic segments 1-5 $\left(\mathrm{T}_{1}-\mathrm{T}_{5}\right)$ through dorsal root ganglia (DRG), thereby ascending to higher brain regions (3). In patients with chronic cardiac pain, increased activity is found in the insular cortex, believed to be a pivitol region that modulates neuropathic pain $(4,5)$. CMI rats show potentiated synaptic transmission in the nucleus of the solitary tract (NTS) which strongly indicates sensitization, an important phenomenon that occurrs in neuropathic pain as well $(6,7)$. Consequently, cardiac pain in CMI shares similarities with neuropathic pain (5). Moreover, glial activation has been demonstrated to be involved in the spinal cord under neuropathic pain conditions (7). However, to the best of our knowledge, whether spinal glial cells are involved in chronic cardiac pain in CMI has not been previously investigated.

Spinal cord stimulation (SCS) is an alternative therapeutic approach that has been clinically utilized for decades to treat patients with CMI, providing an improvement in intractable 
angina symptoms $(8,9)$. SCS owes its inception to the classical 'gate-control' theory, in which the selective stimulation of non-nociceptive afferent fibers $(A \beta)$ inhibits nociceptive afferent fibers (A $\delta$ and $\mathrm{C}$ ) through the activation of inhibitory interneurons in the substantia gelatinosa of the spinal dorsal horns (10). Although this mechanism may explain the immediate and short-term action of SCS, it does not readily account for the prolonged analgesic effect, which may reflect a potential anti-central sensitization mechanism by SCS in neuropathic pain. A previous study suggested that SCS could reduce mechanical hyperalgesia by inhibiting spinal glial activation in rats with neuropathic pain (11). Therefore, we hypothesized that SCS may produce a cardiac analgesic effect partially by inhibiting the activation of spinal glia in CMI model rats. The current study tested this hypothesis directly in model rats with CMI induced by left anterior descending artery (LAD) ligation to produce cardiac pain (6).

\section{Materials and methods}

Animals. Experiments were performed on male adult Sprague-Dawley rats (age, 12 weeks; weight, 250-300 g; purchased from the Laboratory Animals Center of the General Hospital of Western Command Theater; $n=88$ ), housed in transparent plastic cages at $22-25^{\circ} \mathrm{C}$ and $55 \pm 5 \%$ relative humidity with free access to food and water, under a 12-h light/dark cycle. Rats were acclimated to the observation room for $30 \mathrm{~min}$ prior to all behavioral experiments. Rats were randomly separated into eight groups: Naive group $(\mathrm{n}=8)$, sham-operated group $(\mathrm{n}=16)$, CMI group $(\mathrm{n}=16)$, CMI+SCS group $(n=16), C M I+v e h i c l e(V e h)$ group $(n=8)$, $\mathrm{CMI}+$ minocycline (Mino) group $(\mathrm{n}=8), \mathrm{CMI}+\mathrm{SCS}+\mathrm{Veh}$ group $(\mathrm{n}=8)$, CMI+SCS+fractalkine (Frac) group $(\mathrm{n}=8)$. Animal experiments were performed in accordance with the ethical guidelines of the International Association for the Study of Pain (12) and were approved by the Animal Use and Care Committee for Research and Education at the Air Force Military Medical University and General Hospital of Western Command Theater (Chengdu, China; approval no. 10070). Rats were anesthetized with an intraperitoneal (i.p.) injection of pentobarbital $(60 \mathrm{mg} / \mathrm{kg})$ prior to operation. At the end of the study, rats were euthanized with an overdose of pentobarbital $(100 \mathrm{mg} / \mathrm{kg}$; i.p.). All efforts were made to minimize animal suffering and the number of animals used.

Establishment of the CMI model. LAD ligation was performed to produce a CMI rat model based on a previously described procedure (13). Briefly, rats were anesthetized with pentobarbital $(60 \mathrm{mg} / \mathrm{kg}$; i.p.) and, after endotracheal intubation and assisted ventilation (tidal volume, $4 \mathrm{ml}$; ventilation rate, $60 / \mathrm{min}$ ), the heart was exposed through a left thoracotomy at the level of the fifth intercostal space. The LAD was then occluded $3 \mathrm{~mm}$ from its origin with a 6-0 prolene suture. The chest was then closed, and each rat was allowed to recover before the subsequent experiments. Sham-operated rats underwent an identical surgery but without LAD ligation. Successful establishment of the CMI model was confirmed by observing elevation of the ST-segment on electrocardiography and pale discoloration of the myocardium post-mortem.
$S C S$. After LAD ligation, a laminectomy was performed at the level of $\mathrm{T}_{5}$ vertebra, and the SCS lead was inserted epidurally in the rostral direction. The lead was fixed with sutures to the muscle, the wound was sutured in layers, and the lead was tunneled to exit the skin at the base of the neck. A spinal cord lead designed for use in rats (Medtronic plc) that is similar to that used in patients was inserted (11); the proximal end of the lead was tunneled outside the animal for later connection to an external neurostimulator (model no. 37021) and programmer (model no. 8840; both Medtronic plc). Based on a previous study (6), $60 \mathrm{~Hz} \mathrm{SCS}$ at $90 \%$ motor threshold (amplitude; $0.35 \mathrm{~V}$ ) was chosen in the current study. The duration of SCS in the present study is shown in Fig. 1.

\section{Behavioral experiments}

Open field $(O F)$ test. Rats were placed in an OF $\left(100 \times 100 \times 40 \mathrm{~cm}^{3}\right)$ inside a dimly lit isolation chamber $(<50$ lux in the center of the open field) with a fan. An activity-monitoring system (Panlab) was used to record horizontal locomotor activity. Briefly, this system used paired sets of photobeams to detect movement in the open field and movement was recorded as beam breaks. Each animal was placed in the center of the OF box, and its vertical (rearing; rats standing up on their hindlimbs) activity and distance traveled were recorded and measured for $15 \mathrm{~min}$. Fewer rearing motions of the rats reflected more severe spontaneous pain conditions, and vice versa (14). SCS was applied 30 min daily for 4 consecutive days before the OF test (Fig. 1A).

Conditioned place preference (CPP) test. A multi-trial conditioning protocol was used for CPP, as previously described (15). Preconditioning to an automated three-chamber CPP box was performed for 3 days, starting 10 days after CMI surgery (D10-D12). All animals were acclimated with full access to all chambers for 30 min each day. On D12, behavior was recorded for $15 \mathrm{~min}$ and analyzed to confirm the absence of a preference for a particular preconditioning chamber. Following the preconditioning phase, the rats underwent conditioning for 8 days with alternating SCS-treatment chamber pairings. Rats received non-SCS-chamber pairing (rats were tethered to the neurostimulator system but did not receive SCS) on odd days (D13, 15, 17 and 19) and SCS-chamber pairing on even days (D14, 16, 18 and 20). Rats were placed in the paired chamber with no access to the other chamber following non-SCS or SCS. SCS and chamber pairings were counterbalanced. The conditioning time was $30 \mathrm{~min}$ in each chamber. On the testing day (D21), rats were placed into the neutral chamber of the CPP box with free access to all chambers and their behavior was recorded for $15 \mathrm{~min}$ to analyze their chamber preference (Fig. 1B). Increased time spent in a chamber (for example, increased time in the SCS-paired chamber) indicated a preference for that chamber, suggesting a pain-relieving effect (analgesia) (15).

Immunofluorescence and Masson's trichrome staining. Immunofluorescence staining was performed as previously described (16). The rats were deeply anesthetized with an overdose of pentobarbital (100 mg/kg; i.p.) and transcardially perfused with $150 \mathrm{ml} 0.01 \mathrm{M}$ PBS (pH 7.4), followed by $500 \mathrm{ml}$ $4 \%$ paraformaldehyde in $0.1 \mathrm{M}$ phosphate buffer ( $\mathrm{pH}$ 7.4). The 

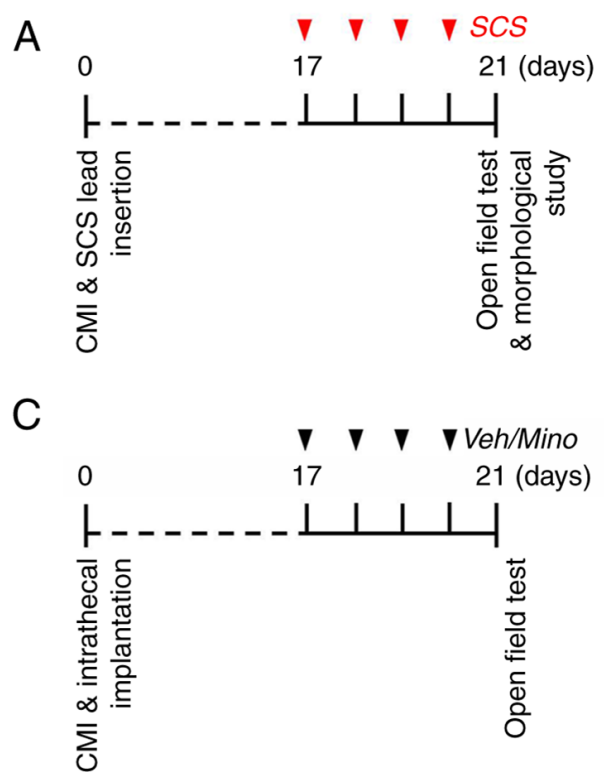

B
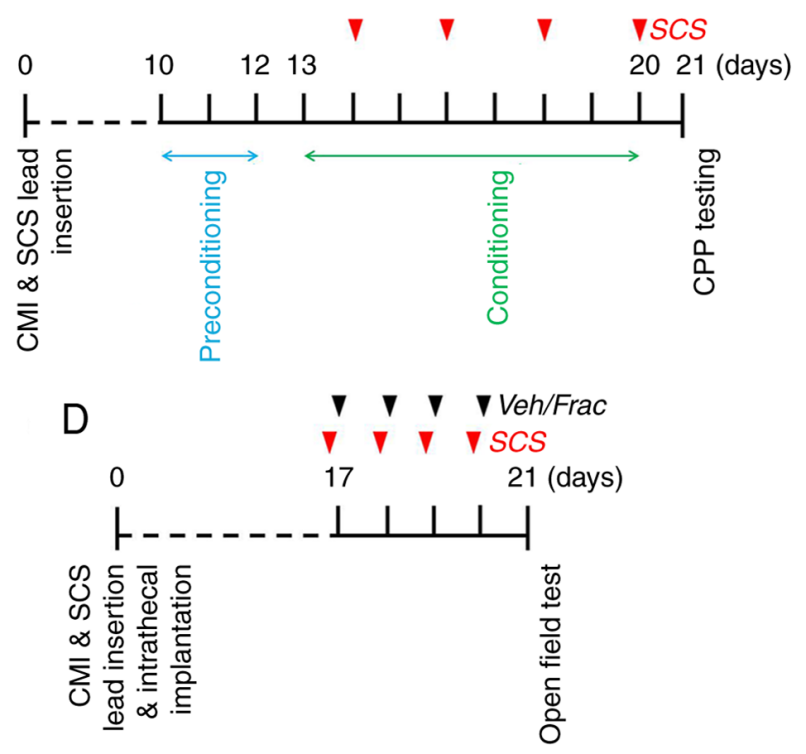

Figure 1. Experimental schedule. Timeline of SCS treatment, intrathecal implantation, drug administration and behavioral tests after CMI. (A) SCS was administered once a day from day 17 to 20 after CMI. Open field test and a morphological study were conducted at 21 days after CMI surgery. (B) Preconditioning and conditioning were performed from days 10 to 20, followed by CPP testing on day 21. (C) Mino and (D) Frac or vehicle were intrathecally administered daily from days 17 to 20 after CMI. The open field test was performed on day 21. CMI, chronic myocardial ischemia; CPP, conditioned place preference; frac, fractalkine; mino, minocycline; SCS, spinal cord stimulation; veh, vehicle.

heart was harvested and embedded in paraffin. The embedded heart was sectioned at $6 \mu \mathrm{m}$ and Masson's trichrome staining kit (cat. no. HT15; Sigma-Aldrich; Merck KGaA) was used according to the manufacturer's protocol. Masson's trichrome staining was performed to verify myocardial fibrosis induced by CMI at 3 weeks post-surgery. The spinal cord was transversely sliced into $25-\mu \mathrm{m}$-thick coronal sections using a freezing microtome (CM1950; Leica Microsystems GmbH).

Double-immunofluorescence staining for i) ionized calcium-binding adaptor protein-1 (Iba-1; microglial marker) and phosphorylated (p)-p38, ii) glial fibrillary acidic protein (GFAP; astrocyte marker) and p-p38, or iii) neuronal nuclei (NeuN; neuronal marker) and p-p38 was performed; the antibodies used are shown in Table I. The spinal cord sections were sequentially incubated at room temperature with primary antisera in 0.01 M PBS containing 5\% normal donkey serum (NDS, cat. no. 566460; MilliporeSigma), 0.3\% Triton X-100, $0.05 \% \mathrm{NaN}_{3}$ and $0.25 \%$ carrageenan (PBS-NDS; $\mathrm{pH} 7.4$ ) for $24 \mathrm{~h}$. Then, the sections were incubated with the corresponding Alexa 488 or 594-conjugated secondary antibodies for $6 \mathrm{~h}$ at room temperature. A negative control experiment, in which the primary antibodies were omitted, and a peptide competition assay were performed. No immunopositive products were detected.

After the immunofluorescence staining, the sections were observed and images were captured using a confocal laser-scanning microscope (FV1000; Olympus Corporation) using FLUOVIEW software (FV10-ASW 1.7 Viewer; Olympus Corporation). In total, five non-adjacent sections from the $\mathrm{T}_{1}-\mathrm{T}_{5}$ segments were selected at random per rat $(n=8)$. Images were evaluated using a computer-assisted image analysis program (MetaMorph 6.1; Molecular Devices, LLC) which set the low and high thresholds for the immunofluorescent intensity that was determined to be a signal. Imaging data were collected using the same region and the same size of field within the spinal dorsal horn. The same configuration was used to measure cell areas in all experimental groups. The measured areas were transferred to Excel (Microsoft Corporation) automatically for the statistical analysis. MetaMorph 6.1 was calibrated to provide standardization of area measurements. The immunoreactivities for GFAP and Iba-1 within the superficial dorsal horn were averaged across the five spinal sections for the experimental group (17).

Western blotting. Rats were deeply anesthetized with an overdose of pentobarbital (100 mg/kg; i.p.), and the $\mathrm{T}_{1}-\mathrm{T}_{5}$ segments of the spinal dorsal cord were carefully dissected and harvested for western blotting. To obtain total protein extracts, the tissues were lysed in $300 \mu \mathrm{l}$ lysis buffer containing $10 \mathrm{mM}$ Tris, $150 \mathrm{mM} \mathrm{NaCl}, 1 \%$ Triton X-100, 0.5\% NP-40 and $1 \mathrm{mM}$ EDTA at $\mathrm{pH}$ 7.4. The samples were adequately mixed with protease inhibitor cocktail and phosphatase inhibitor cocktail (Roche Diagnostics) at a 100:1 (v/v) ratio. Subsequently, $30 \mu \mathrm{g}$ cell lysis material (quantitatively measured using the BCA protein assay; Thermo Fisher Scientific, Inc.) was resolved by SDS-PAGE (10\% SDS-polyacrylamide gels) and transferred to PVDF membranes (Immobilon-P; MilliporeSigma). After blocking in $3 \%$ non-fat milk for $1 \mathrm{~h}$ at room temperature, the membranes were incubated overnight at $4{ }^{\circ} \mathrm{C}$ with the following primary antibodies: Rabbit anti-p38 (1:1,000; cat. no. 8690; Cell Signaling Technology, Inc.) rabbit anti-p-p38 (1:1,000; cat. no. 4511; Cell Signaling Technology, Inc.) and rabbit anti-GAPDH (1:1,000; cat. no. 2118; Cell Signaling Technology, Inc.). The immunoblots were then incubated with an HRP-conjugated goat anti-rabbit secondary antibody (1:5,000; cat. no. ER48616; Amersham; Cytiva) at room temperature for $2 \mathrm{~h}$. All of the reactions were detected using the ECL detection method (Amersham; Cytiva) and 
Table I. Antisera used in each group.

\begin{tabular}{|c|c|c|}
\hline Group & Primary antibody & Secondary antibody \\
\hline NeuN/p-p38 & $\begin{array}{l}\text { Mouse anti-NeuN (1:500; cat. no. ZMS377; } \\
\text { MilliporeSigma) } \\
\text { Rabbit anti-p-p38 (1:200; cat. no. 4511; } \\
\text { Cell Signaling Technology, Inc.) }\end{array}$ & $\begin{array}{l}\text { Alexa } 488 \text { Donkey anti-mouse (1:500; cat. no. A-11001; } \\
\text { Invitrogen; Thermo Fisher Scientific, Inc.) } \\
\text { Alexa } 594 \text { Donkey anti-rabbit (1:500; cat. no. R37119; } \\
\text { Invitrogen; Thermo Fisher Scientific, Inc.) }\end{array}$ \\
\hline GFAP/p-p38 & $\begin{array}{l}\text { Mouse anti-GFAP (1:500; cat. no. SAB5201104; } \\
\text { MilliporeSigma) and } \\
\text { rabbit anti-p-p38 (1:200; cat. no. 4511; } \\
\text { Cell SignalingTechnology, Inc.) }\end{array}$ & $\begin{array}{l}\text { Alexa } 488 \text { Donkey anti-mouse (1:500; cat. no. A-11001; } \\
\text { Invitrogen; Thermo Fisher Scientific, Inc.) and } \\
\text { Alexa 594Donkey anti-rabbit (1:500; cat. no. R37119; } \\
\text { Invitrogen; Thermo Fisher Scientific, Inc.) }\end{array}$ \\
\hline Iba-1/p-p38 & $\begin{array}{l}\text { Goat anti-Iba-1 (1:500, cat. no. 011-27991; } \\
\text { Wako) } \\
\text { Rabbit anti-p-p38 (1:200, cat. no. } 4511 \text {; } \\
\text { Cell Signaling Technology) }\end{array}$ & $\begin{array}{l}\text { Alexa } 488 \text { Donkey anti-goat (1:500; cat. no. A-11055; } \\
\text { Invitrogen; Thermo Fisher Scientific, Inc.) } \\
\text { Alexa } 594 \text { Donkey anti-rabbit (1:500; cat. no. R37119; } \\
\text { Invitrogen; Thermo Fisher Scientific, Inc.) }\end{array}$ \\
\hline
\end{tabular}

GFAP, glial fibrillary acidic protein; Iba-1, ionized calcium-binding adaptor protein-1; NeuN, neuronal nuclei; p-, phosphorylated.

Table II. Primers sequences used for reverse transcription-quantitative PCR.

\begin{tabular}{llr}
\hline Gene & \multicolumn{1}{c}{ Primer sequence $\left(5^{\prime} \rightarrow 3^{\prime}\right)$} & Accession no. \\
\hline TNF- $\alpha$ & F: TGATCGGTCCCAACAAGG A & AY427675 \\
IL-1 $\beta$ & R: TGCTTGGTG GTTTGCTACGA & NM031512 \\
GAPDH & F: TGCTGATGTACCAGTTGGGG & NM01008 \\
& R: CTCCATGAGCTTTGTACAAG & F: CCCCCAATGTATCCGTTGTG \\
\hline
\end{tabular}

F, forward; R, reverse.

exposure to film. The scanned images were semi-quantified and analyzed with ImageJ $1.52 \mathrm{v}$ software (National Institutes of Health). A square of the same size was drawn around each band to measure the density, and the background near that band was subtracted. The density of specific p-p38 bands was measured and normalized against total p38 expression.

Reverse transcription-quantitative PCR (RT-qPCR). RT-qPCR was performed in as previously described (18). Rats were anesthetized with sodium pentobarbital (100 mg/kg; i.p.), the $\mathrm{T}_{1}-\mathrm{T}_{5}$ spinal dorsal horn was rapidly harvested, and total RNA was extracted with TRIzol ${ }^{\circledR}$ (Invitrogen; Thermo Fisher Scientific, Inc.). In accordance with the manufacturer's protocols, cDNA was synthesized with oligo $(\mathrm{dT})_{12-18}$ using Superscript ${ }^{\mathrm{TM}}$ III reverse transcriptase for RT-PCR (Invitrogen; Thermo Fisher Scientific, Inc.). The primers used in the present study are shown in Table II. Equal amounts of RNA were used to prepare cDNA using SYBR Premix Ex Taq (Takara Bio, Inc.) and analyzed by qPCR (Applied Biosystems; Thermo Fisher Scientific, Inc.). The amplification protocol was as follows: Initial denaturation for $3 \mathrm{~min}$ at $95^{\circ} \mathrm{C}$; followed by 45 cycles of denaturation for $10 \mathrm{sec}$ at $95^{\circ} \mathrm{C}$ and annealing and extension for $45 \mathrm{sec}$ at $60^{\circ} \mathrm{C}$. Target cDNA quantities were estimated from the quantification amplification cycle number $(\mathrm{C} 1)$ using
Sequence Detection System software (Applied Biosystems; Thermo Fisher Scientific, Inc.). A ${ }^{\Delta} \mathrm{Cq}$ value was calculated for each sample by subtracting its $\mathrm{Cq}$ value from the $\mathrm{Cq}$ value for the corresponding GAPDH to normalize the differences in cDNA aliquots. Each cDNA quantity was then calculated with the following formula: $2^{\Delta} \mathrm{Cq}(19)$.

Intrathecal catheter implantation and drug administration. Intrathecal catheter implantation surgery was performed immediately following CMI or SCS operation under pentobarbital anesthetization. It was performed by inserting polyethylene tubing through which the drug (minocycline, fractalkine, or vehicle as mentioned below) was directly injected into the subarachnoid space of the thoracic segment following CMI or SCS operation. The catheters were primed with vehicle (sterile saline solution, cat. no. BTYY1000T; Nanjing Bianzhen Biological Technology) before implantation and the neck incision was closed with sutures. The catheter was left in place until the required experiments had been completed. At the end of each experiment, the position of the polyethylene tubing in the intrathecal space was visually confirmed by exposing the spinal cord post-mortem.

To investigate the involvement of microglial activation in the CMI-induced cardiac pain, the following behavioral 
pharmacological experiments were performed. Intrathecal (i.t.) administration of minocycline (100 $\mu \mathrm{g} / 10 \mu \mathrm{l}$; cat. no. M9511; Sigma-Aldrich; Merck KGaA) was selected to inhibit microglial activation, in accordance with a previous report (20). Fractalkine (60 ng/10 $\mu$ i; i.t.; cat. no. F135; Sigma-Aldrich; Merck $\mathrm{KGaA}$ ), a microglia-activating factor, was used to induce microglial activation (21). After CMI establishment, minocycline or vehicle was applied daily from D17 to D20. OF tests were then performed to verify the effects of the drug on cardiac pain behaviors of CMI model rats (Fig. 1C). In CMI model rats receiving SCS treatment, either vehicle or fractalkine was administered intrathecally $1 \mathrm{~h}$ after SCS to detect the reversing influence of fractalkine on the SCS-produced analgesia by OF testing (Fig. 1D).

Statistical analysis. Statistical analyses were performed using SPSS 22.0 software (IBM, Corp.). The results are expressed as the mean \pm SEM. Two-way ANOVA with Bonferroni multiple-comparison tests or one-way ANOVA with Tukey's multiple-comparison post hoc tests were used for between-group comparisons. For CPP experiments, data were analyzed before conditioning (baseline) and after conditioning using two-factor ANOVA (chambers vs. treatment) followed by Student's t-test with Bonferroni correction. $\mathrm{P}<0.05$ was considered to indicate a statistically significant difference.

\section{Results}

CMI established by LAD ligation produces significant spontaneous cardiac pain in rats. CMI model establishment was confirmed by examining a remote area of the myocardium becoming pale after the LAD ligation. Chronic infarction was confirmed using Masson's trichrome stain to label collagen scar tissue (blue) and cardiac muscle (red) 3 weeks post-operation (Fig. 2). No difference was found between the naive and sham groups of rats; however, clear collagen scarring rather than normal cardiac muscle tissue was observed at cross sections of ventricular papillary muscles in CMI model rats, in contrast to the findings in naive and sham rats (Fig. 2), indicating successful establishment of the CMI model.

Cardiac pain induced by CMI belongs to visceral pain (3). Thus, OF tests were performed to further observe the spontaneous pain behaviors based on previous reports $(14,22)$. The current study evaluated the rearing count and distance traveled, which could reflect visceral pain. No statistically significant differences in either vertical count or distance traveled were observed between the naive and sham-operated groups $(n=8$; Fig. 3A and B, respectively). However, the vertical count was significantly decreased in CMI model rats on day 21 post-operation compared with that in the sham group ( $n=8$; Fig. 3A). The results demonstrated that CMI established by LAD ligation produced pronounced spontaneous cardiac pain. Similarly, the distance traveled was also greatly decreased in CMI model rats compared with that in the sham group ( $n=8$; Fig. 3B), indicating that locomotion was negatively affected by LAD ligation owing to cardiac insufficiency in CMI model rats.

SCS effectively alleviates cardiac pain in CMI model rats. Based on several studies on animals and human patients,

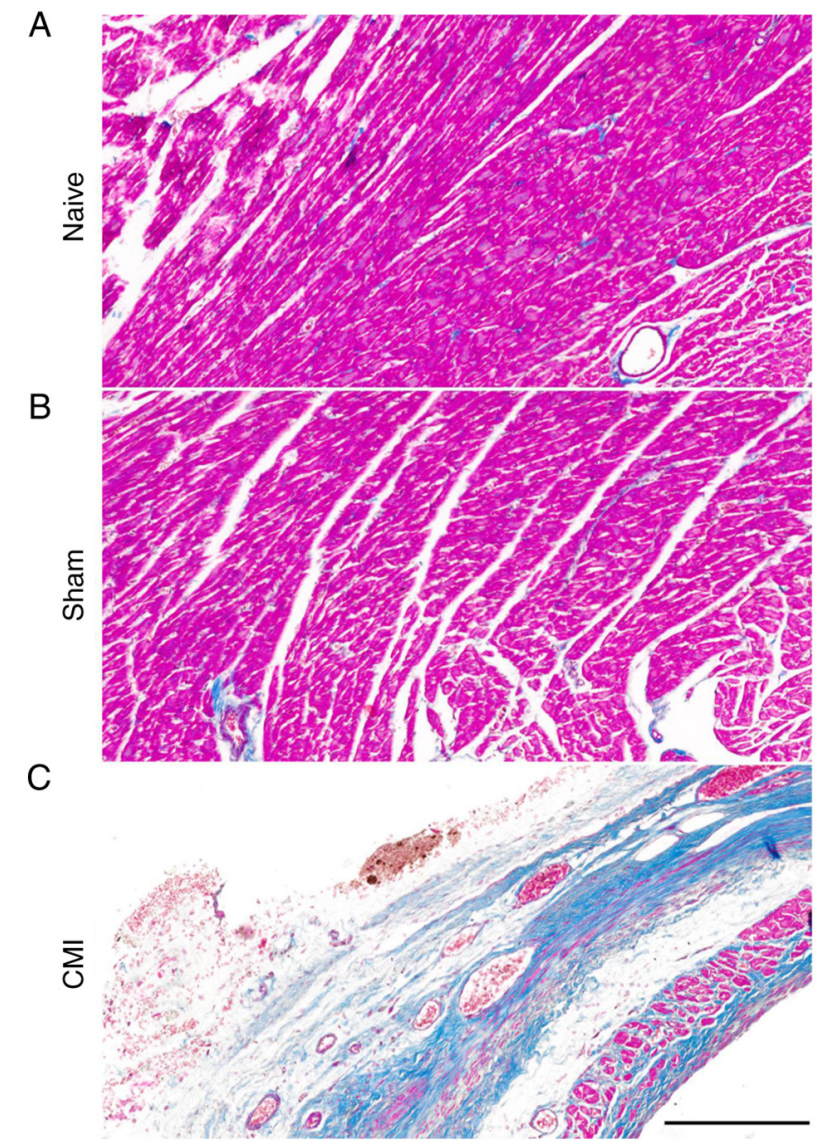

Figure 2. Representative images from Masson's trichrome staining in heart tissues from the different groups. Chronic infarct size in (A) naive, (B) sham and (C) CMI rats was determined using Masson's trichrome stain of heart tissue at the border zone of infarcted myocardium at cross sections of ventricular papillary muscles to label collagen scar tissue (blue) and cardiac muscle (red) 21 days after left anterior descending artery ligation surgery. An increased collagen area was observed in the CMI group. Scale bar, $100 \mu \mathrm{m}$. CMI, chronic myocardial ischemia.

SCS is considered to be an effective alternative therapy to treat many types of intractable pain, especially chronic pain $(23,24)$. Thus, it was next determined whether SCS could alleviate such spontaneous cardiac pain in CMI model rats. Before OF testing, SCS was performed on 4 consecutive days in CMI model rats (Fig. 1A). Interestingly, SCS improved the spontaneous cardiac pain, as revealed by an increasing number of vertical counts in CMI model rats compared with untreated CMI rats (n=8; Fig. 3A). However, there was no significant difference between CMI and CMI + SCS groups in terms of locomotion, as revealed by the distance traveled during the 15 -min recording time in the $\mathrm{OF}$ test $(\mathrm{n}=8$; Fig. $3 \mathrm{~B})$. The findings suggested that the effects of SCS on increasing vertical counts did not depend on an improved locomotor ability in CMI model rats. Thus, SCS could effectively alleviate the spontaneous pain in CMI model rats without changing the declined locomotor abilities.

It has been shown that chronic visceral pain produces not only spontaneous pain but also ongoing pain conditions (25). Thus, a CPP test was conducted to investigate the effects of SCS on ongoing pain in CMI model rats. Following a 3-day preconditioning phase, rats underwent conditioning (8 days) using SCS or non-SCS treatment paired with alternate 

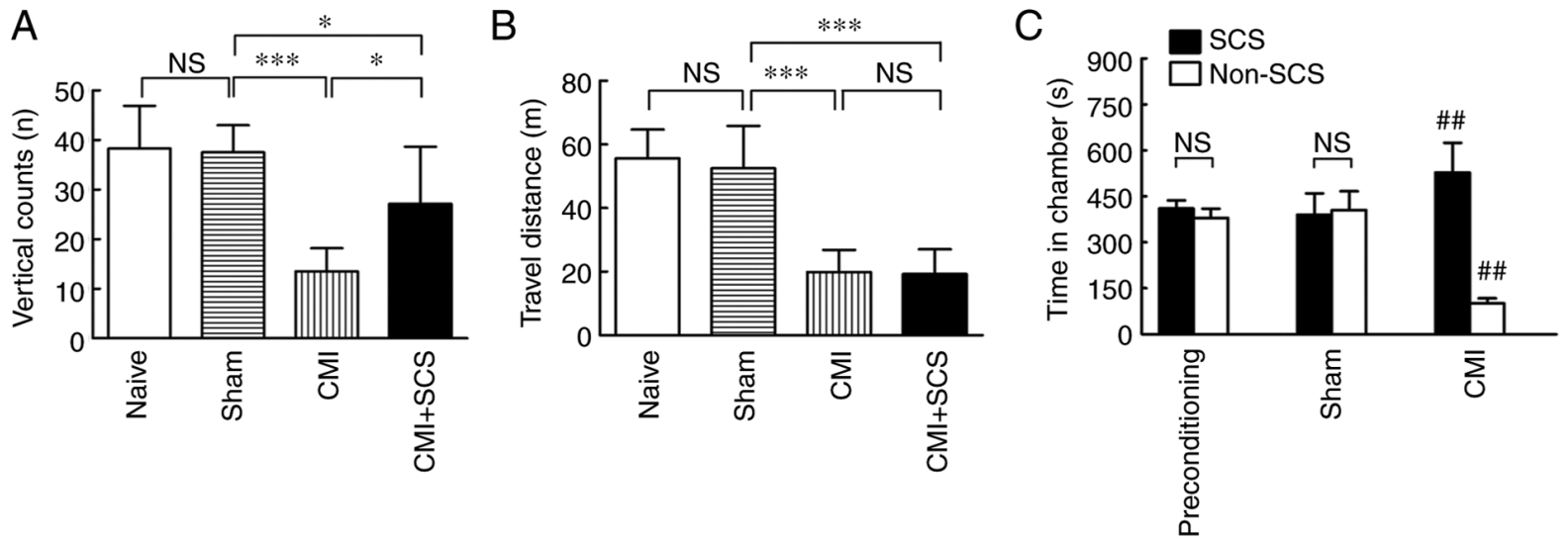

Figure 3. Behavioral assessments of rats in open field and conditioned place preference tests. (A) Vertical counts and (B) travel distance were decreased in rats with CMI in the open field test. SCS significantly increased the vertical counts but did not affect the distance traveled in CMI model rats. (C) Conditioning with SCS increased the time spent in the paired chamber of rats with CMI, but not in sham-operated rats, with a corresponding decrease in the non-SCS-paired chamber. $\mathrm{N}=8$ rats/group; ${ }^{*} \mathrm{P}<0.05,{ }^{* * * *} \mathrm{P}<0.001 ;{ }^{\# \#} \mathrm{P}<0.01 \mathrm{v}$. pre-conditioning values. NS, not significant; CMI, chronic myocardial ischemia; SCS, spinal cord stimulation.

chambers on alternate days (Fig. 1B). On the testing day, rats were placed in the CPP box with free access to all of the chambers. Only CMI model rats showed an increase in the time spent in the SCS-paired chambers ( $n=8$; Fig. 3C). All groups spent equivalent time in the neutral chamber. SCS did not produce any preference in the sham-operated rats, indicating that it was not beneficial in the absence of CMI. The finding that SCS produced CPP in CMI model rats demonstrated that SCS could improve the ongoing pain under CMI conditions.

SCS inhibits microglial but not astrocyte activation via the p-p38 pathway in spinal cord of CMI model rats. Spinal cord glial cells have been reported to be involved in the induction and maintenance of chronic pain (26). Therefore, glial activation in the spinal cord of CMI model rats after SCS treatment was examined. Results from immunofluorescence staining revealed that the integrated densities of Iba-1-labeled microglia, as well as GFAP-labeled astrocytes, were significantly increased in the $\mathrm{T}_{1}-\mathrm{T}_{5}$ spinal dorsal horn 3 weeks after LAD ligation (Fig. 4). However, SCS could effectively suppress spinal dorsal horn Iba-1 upregulation (Fig. 4H), but not that of GFAP in CMI model rats (Fig. 4G), which indicated that SCS could inhibit CMI-induced microglia instead of astrocyte activation during the maintenance of chronic cardiac pain.

As previous studies reported that functional microglial activation was associated with the phosphorylation of p38 MAPK $(21,27)$, double-immunofluorescence staining for spinal p-p38 was performed in rats with CMI. As shown in Fig. 5, the immunoreactivity for p-p38 was exclusively colocalized with Iba-1-positive cells but not with NeuN-positive (neuronal marker) or GFAP-positive ones (astrocyte marker). These results suggested that spinal dorsal horn p-p38 expression may be a specific downstream signaling pathway in spinal dorsal microglia after CMI.

It was also observed that SCS could effectively inhibit the upregulation of p-p38 expression in CMI model rats (Fig. 6A-D). Subsequently, western blotting was conducted to further analyze p-p38 expression in the thoracic spinal dorsal horn in the different groups. Increased ratios of $\mathrm{p}-\mathrm{p} 38 / \mathrm{p} 38$ were noted in rats with CMI compared with those in the sham group (Fig. 6E and F). However, the ratio of p-p38/p38 from the CMI + SCS group was significantly decreased compared with that in the CMI group (Fig. 6E and F). These results suggested that the p-p38 signaling pathway was activated after CMI, and SCS could effectively inhibit this pathway and microglial activation.

Immune responses (glial activation and cytokine release) in the spinal dorsal horn may serve essential roles in the induction and maintenance of chronic pain (28). Thus, the changes in expression levels of pro-inflammatory mediators, including IL-1 $\beta$ and TNF- $\alpha$, in the spinal cord were determined. Using RT-qPCR, it was observed that compared with sham group, $\mathrm{CMI}$ induced significant increases in the mRNA expression levels of pro-inflammatory mediators in the spinal dorsal horn: IL-1 $\beta$ (332.6 $\pm 146.7 \%$; Fig. 7A) and TNF- $\alpha(149.3 \pm 45.2 \%$; Fig. 7B). However, SCS treatment effectively attenuated the mRNA expression of these mediators: IL-1 $\beta(141.7 \pm 36.1 \%)$ and TNF- $\alpha(113.7 \pm 13.1 \%)$. These findings indicated that SCS may prevent the production of pro-inflammatory mediators in the spinal dorsal horn in CMI model rats.

Microglial inhibition reduces the CMI-induced cardiac pain, whereas microglial activation partially reverses the therapeutic effects of SCS on CMI model rats. To further examine the involvement of microglia in the SCS-produced analgesia in CMI model rats, a behavioral pharmacological study was performed. As shown in Fig. 1C and D, CMI model rats with LAD ligation were simultaneously implanted with an intrathecal catheter. The vertical counts in the OF tests were recorded in the different groups to reflect the pain behaviors. The intrathecal administration of minocycline, a microglial inhibitor, to CMI model rats significantly increased the vertical counts compared with CMI+Veh group (Fig. 8), which indicated that microglial activation may contribute to the CMI-induced cardiac pain. Nonetheless, the vertical activity times in the $\mathrm{CMI}+$ minocycline group remained lower compared with those in the CMI + SCS group. The microglial activator fractalkine was then administered intrathecally to activate spinal cord microglia in CMI model rats with SCS treatment. Interestingly, compared with the vehicle group, the improved cardiac pain behaviors after SCS treatment were in turn aggravated in the fractalkine group, as revealed 

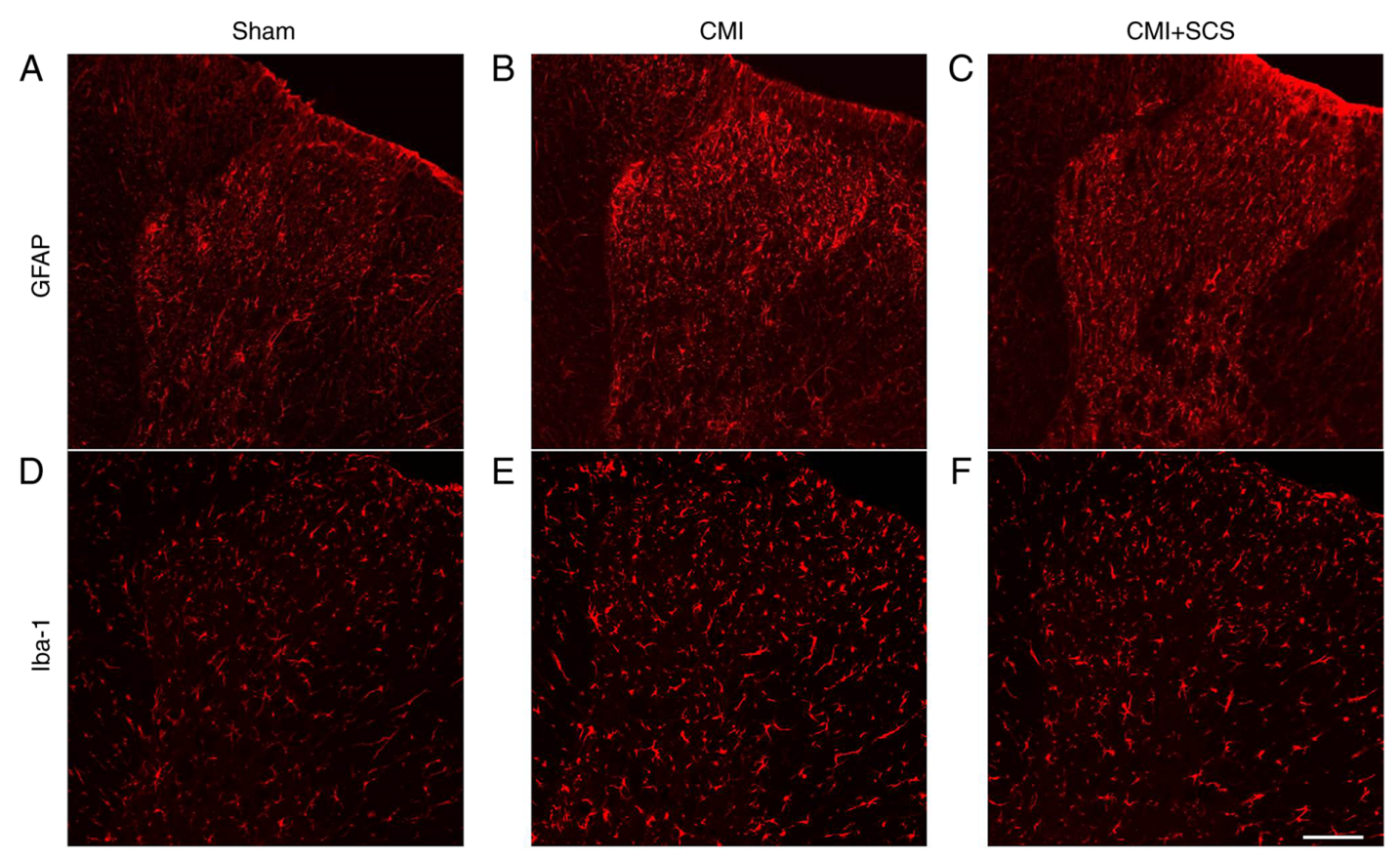

G
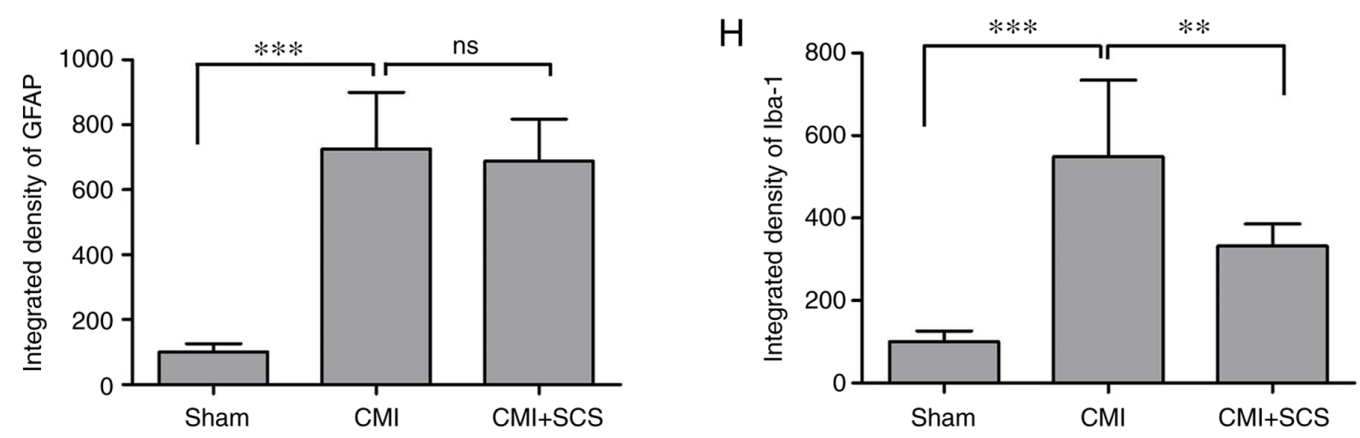

Figure 4. Changes in the immunoreactivity levels of GFAP and Iba-1 in the thoracic spinal cord after SCS in CMI model rats. Representative images of (A-C) GFAP-positive astrocytes and (D-F) Iba-1-positive microglia in the spinal dorsal horn in the different groups. Scale bar, $100 \mu \mathrm{m}$. Immunoreactivities for (G) GFAP and (H) Iba-1 within the dorsal horn were increased in the CMI group compared with those in the sham group. SCS effectively decreased the immunoreactivity for Iba-1 but not that of GFAP. $\mathrm{N}=8$ rats/group; ${ }^{* *} \mathrm{P}<0.01,{ }^{* * *} \mathrm{P}<0.001$. CMI, chronic myocardial ischemia; GFAP, glial fibrillary acidic protein; Iba-1, ionized calcium-binding adaptor protein-1; ns, not significant; SCS, spinal cord stimulation.

by decreased vertical counts in the OF test. These results demonstrated that microglial inhibition improved the cardiac pain behaviors in CMI model rats. By contrast, rescuing the deactivated microglia in the spinal cord could in part reverse the analgesic effects of SCS, suggesting that the SCS-induced cardiac pain-relieving effects were partially exerted by inhibiting microglial activation.

\section{Discussion}

Cardiac pain information is now considered to be transmitted into the brain via two main ascending pathways: Spinal afferents and vagal afferents (3). Cardiac spinal afferents originating from the heart terminate at the $T_{1}-T_{5}$ segments of the spinal cord dorsal horn through DRG and, thus, ascend to higher brain structures. In addition, vagal afferents also convey cardiac nociceptive information to the NTS in the brainstem and then to higher brain regions (5). It is well known that chronic pain, including CMI-induced chronic cardiac pain, shares several similar characteristics, among which central sensitization is the pivotal mechanism that differs from acute pain (7). In total, three key mechanisms have been implicated in central sensitization: i) Alteration in excitatory glutamatergic neurotransmission, ii) loss of tonic inhibitory controls (disinhibition) and iii) glial-neuronal interactions (7). Our previous work reported that the excitatory synaptic transmission in the NTS was potentiated after CMI (6), indicating that central sensitization developed under chronic cardiac pain conditions. However, to the best of our knowledge, whether central sensitization occurs at the spinal cord level after CMI-induced chronic cardiac pain remains to be investigated.

The present study demonstrated that microglia and astrocytes were activated in the thoracic spinal cord dorsal horn 3 weeks after LAD ligation. The intrathecal administration of the specific microglial inhibitor minocycline alleviated cardiac pain behaviors in CMI model rats. These results suggested that microglial and astrocyte activation was involved in the chronic cardiac pain. The present results coincide with previous findings in nerve injury-induced chronic neuropathic pain as well as inflammatory visceral pain $(21,29)$. In fact, spinal microglia have been demonstrated to contribute to the initiation and maintenance of chronic pain, whereas astrocytes participate in the persistence of chronic pain in later phases (7). However, whether the roles of microglia and astrocytes in CMI-induced 

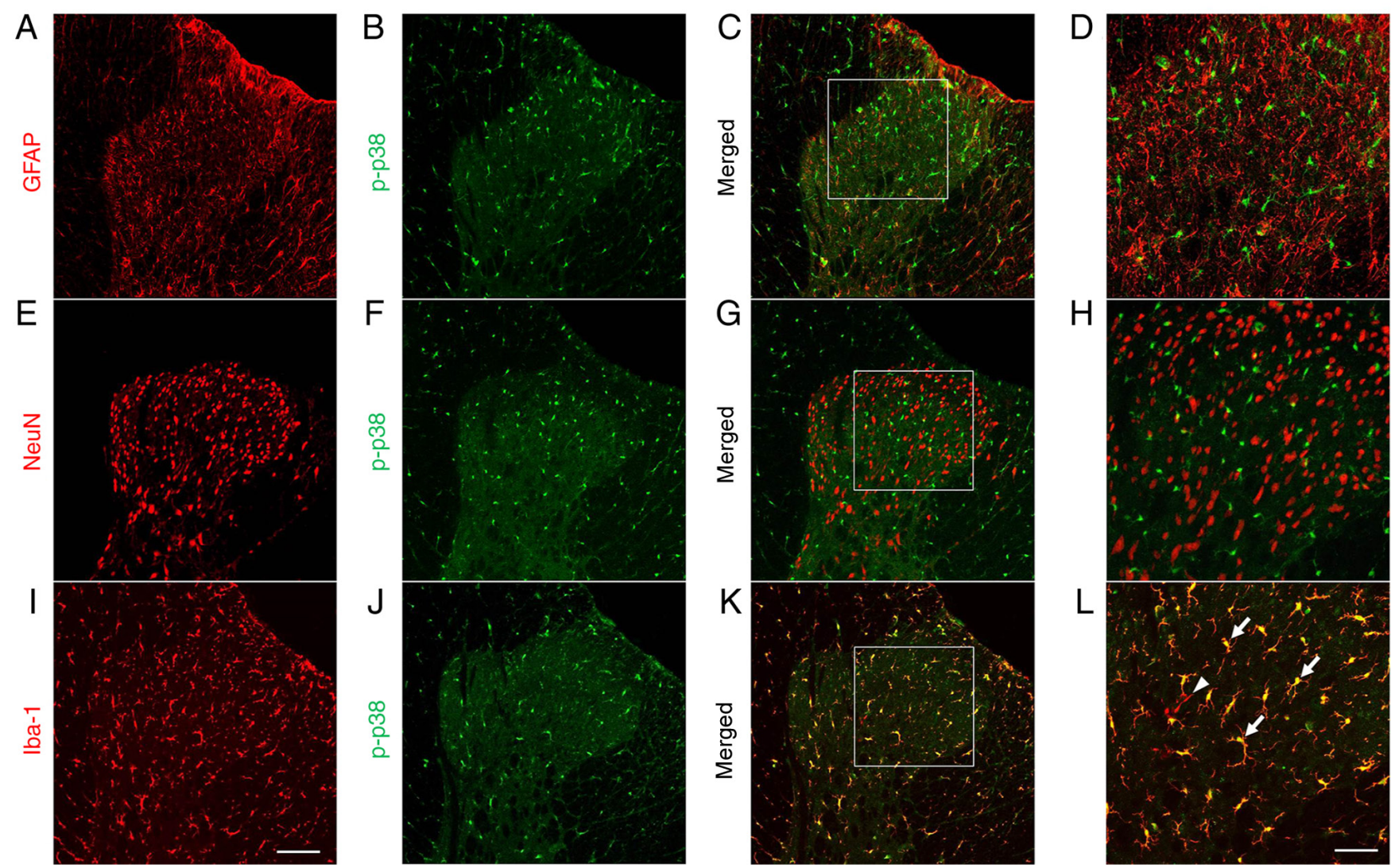

Figure 5. p-p38 MAPK is exclusively expressed in microglia in the spinal cord. Double-immunofluorescence staining showed that spinal p-p38 was not co-expressed with (A-D) GFAP-positive astrocytes or (E-H) NeuN-positive neurons. Instead, p-p38 was colocalized with (I-L) Iba-1-positive microglia. (D, $\mathrm{H}$ and $\mathrm{L}$ ) Magnified images of the rectangles in $(\mathrm{C}, \mathrm{G}$ and $\mathrm{K}$, respectively). Arrows indicate typical double-labeled (yellow) cells and arrowhead indicates Iba-1 single-labeled microglia (red) in panel L. Scale bar, $100 \mu \mathrm{m}$ in panels A-C, E-G and I-K; scale bar, $50 \mu \mathrm{m}$ in panels D, H and L. GFAP, glial fibrillary acidic protein; Iba-1, ionized calcium-binding adaptor protein-1; NeuN, neuronal nuclei; p-, phosphorylated.

cardiac pain are similar to those in neuropathic pain warrants further investigation.

p38 MAPK is activated (phosphorylated) in spinal microglia after nerve injury and is implicated in the production of neuropathic pain (30). A number of studies have also demonstrated that the inhibition of p38 MAPK alleviates allodynia and hyperalgesia in several models of inflammatory and neuropathic pain, suggesting a crucial role of this signaling pathway in pain processing $(31,32)$. In a chronic pancreatitis pain model, spinal p-p38 expression was found to be greatly elevated at 3 weeks after the establishment of pancreatitis and was reversed by i.t. minocycline treatment (21). The current study demonstrated that spinal p-p38 expression was exclusively colocalized with microglia and was significantly increased in CMI model rats. MAPK activation initiates signaling cascades and increases the synthesis of pro-inflammatory mediators. These cytokines can drive central sensitization by increasing excitation in spinal dorsal horn neurons (30). It has been reported that cytokines such as IL-1 $\beta$ released from glial cells could bind to their receptors on neurons and facilitate $\mathrm{N}$-methyl-D-aspartic acid receptor-related synaptic transmission (33). Consistent with this, the current data also suggested that IL- $1 \beta$ and TNF- $\alpha$ were increased in the spinal dorsal horn in CMI model rats, indicating that the microglial p38 MAPK pathway may be activated, and its downstream pro-inflammatory mediators were then elevated under chronic cardiac pain conditions.

Given its efficacy, SCS has been clinically utilized in patients suffering from chronic intractable angina pectoris, especially refractory angina pectoris, as well as cardiac syndrome $\mathrm{X}(2,9)$. It has been reported that SCS could produce an anti-ischemic effect, which was caused by modulation of the sympathetic nervous system, in refractory angina pectoris (34). Moreover, redistribution of coronary blood flow in the heart after SCS was observed in a radiographic study (35). Additionally, echocardiographic studies on both porcine and canine ischemic heart failure models showed that SCS treatment improved left ventricular dilation and cardiac function (36). Although numerous studies on its effects have been performed, the underlying analgesic mechanisms of the cardiac pain-relieving effects of SCS remain to be elucidated. However, accumulating evidence suggests that SCS therapy focuses primarily on neuronal effects (23). Nonetheless, glial cell mechanisms have also been reported to be involved in the SCS-induced analgesia. In a neuropathic pain rat model, SCS was revealed to inhibit spinal glial activation and produce analgesia (11), although the downstream intracellular signaling pathways underlying glial cell modulation induced by SCS were not studied. Moreover, spinal cord glia-related genes were shown to be altered by SCS in nerve injury rats (37). Additionally, SCS was reported to increase M1-like rather than M2-like microglial mRNA in a 

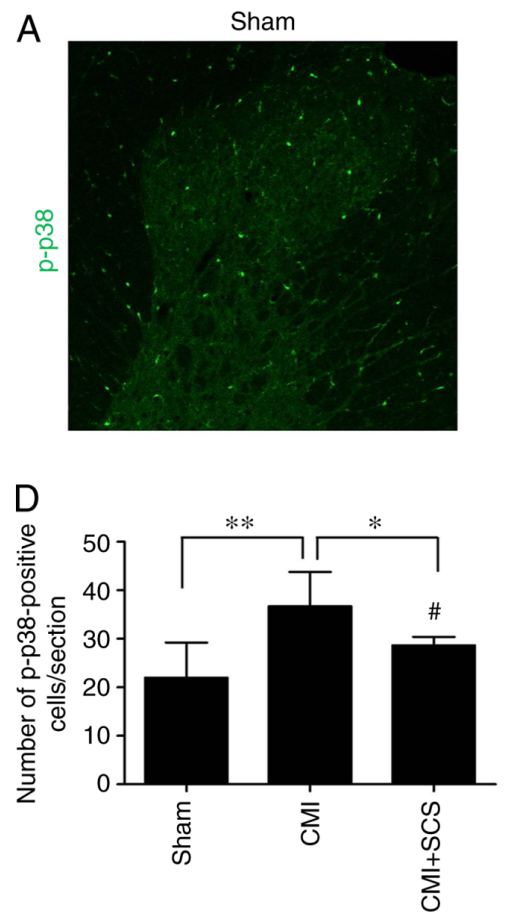

B

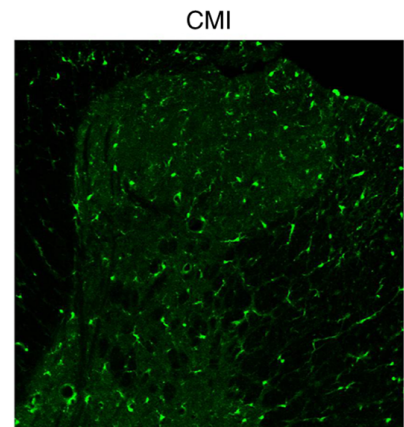

$\mathrm{E}$

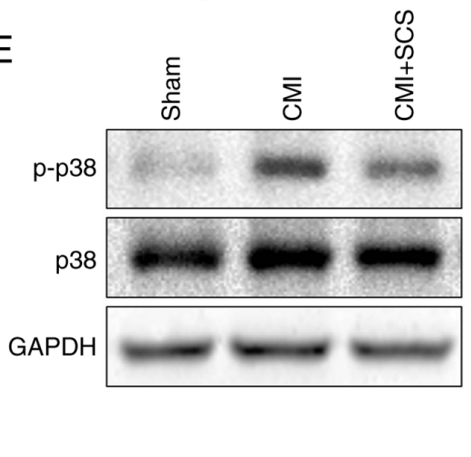

C

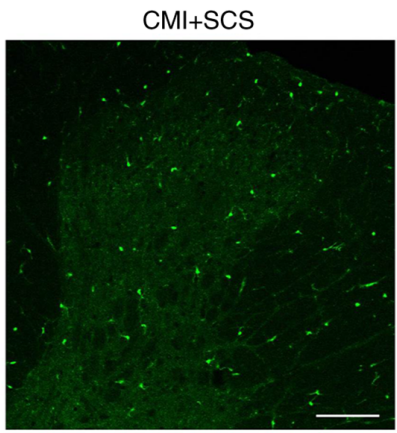

$\mathrm{F}$

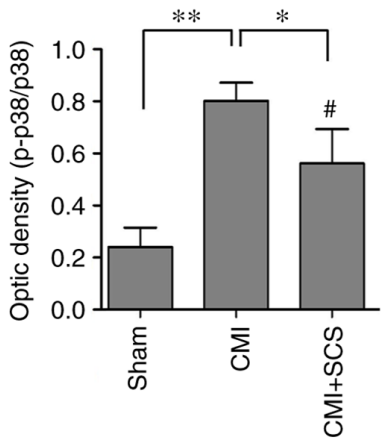

Figure 6. P-p38 upregulation is inhibited by SCS in CMI model rats. Representative immunofluorescence staining images of spinal cord tissue showing the changes of p-p38 expression in (A) sham, (B) CMI and (C) CMI + SCS. Scale bar, $100 \mu \mathrm{m}$. (D) Number of p-p38-positive cells was greatly increased in CMI model rats and was partially inhibited by SCS. (E) Western blotting results were (F) semi-quantified, which indicated that the ratio of p-p38/p38 was elevated in CMI and inhibited by SCS. N=8 rats/group; ${ }^{*} \mathrm{P}<0.05,{ }^{* *} \mathrm{P}<0.01$; ${ }^{*} \mathrm{P}<0.05$ vs. sham. CMI, chronic myocardial ischemia; p-, phosphorylated; SCS, spinal cord stimulation.
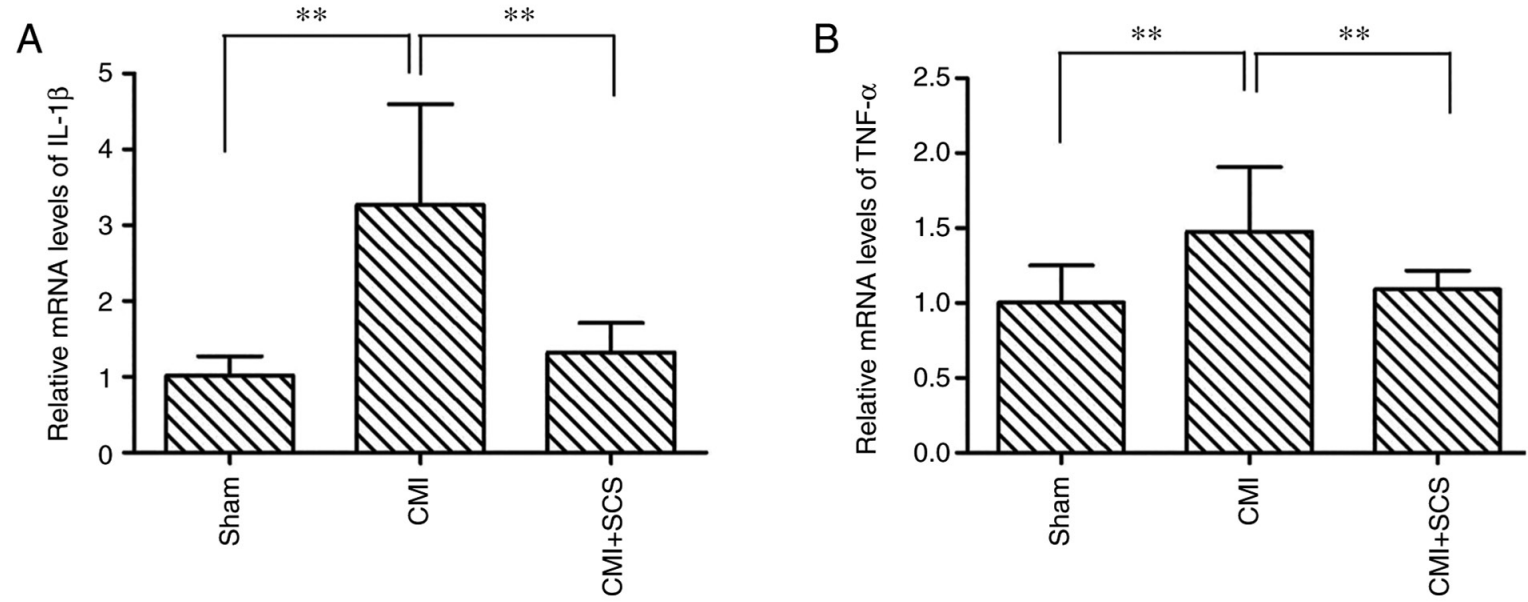

Figure 7. SCS inhibits CMI-induced upregulation of mRNA expression levels of pro-inflammatory mediators. Reverse transcription-quantitative PCR measurement showed that SCS significantly prevented CMI-induced increases in the levels of (A) IL-1 $\beta$ and (B) TNF- $\alpha$ in the spinal dorsal horn. N=8 rats/group; ${ }^{* *} \mathrm{P}<0.01$. CMI, chronic myocardial ischemia; SCS, spinal cord stimulation.

chronic constriction injury model (38). These reports indicate that SCS modulates the activation of glial cells in the spinal cord in neuropathic pain.

In the present work, it was observed that SCS effectively alleviated chronic cardiac pain and inhibited microglial activation in CMI model rats. In addition, SCS inhibited CMI-induced spinal activation of p38 MAPK, which was specifically expressed in microglia rather than astrocytes and neurons. Moreover, the pro-inflammatory mediators IL-1 $\beta$ and TNF- $\alpha$ were downregulated by SCS in CMI model rats. Finally, the activation of microglia partly abolished the SCS-produced pain-relieving effects. These findings suggested that SCS inhibits the microglial p38 MAPK pathway and downstream neuroinflammation, thereby contributing to the alleviation of chronic cardiac pain. The present results agree with previous work that showed SCS decreased microglial expression and p38 MAPK phosphorylation in spared nerve injury-induced neuropathic pain rats (11). Moreover, it has been demonstrated that SCS could reduce the activation of spinal microglia in a spinal cord ischemic reperfusion model in rabbits (39). However, another study suggested that SCS enhanced spinal microglial 


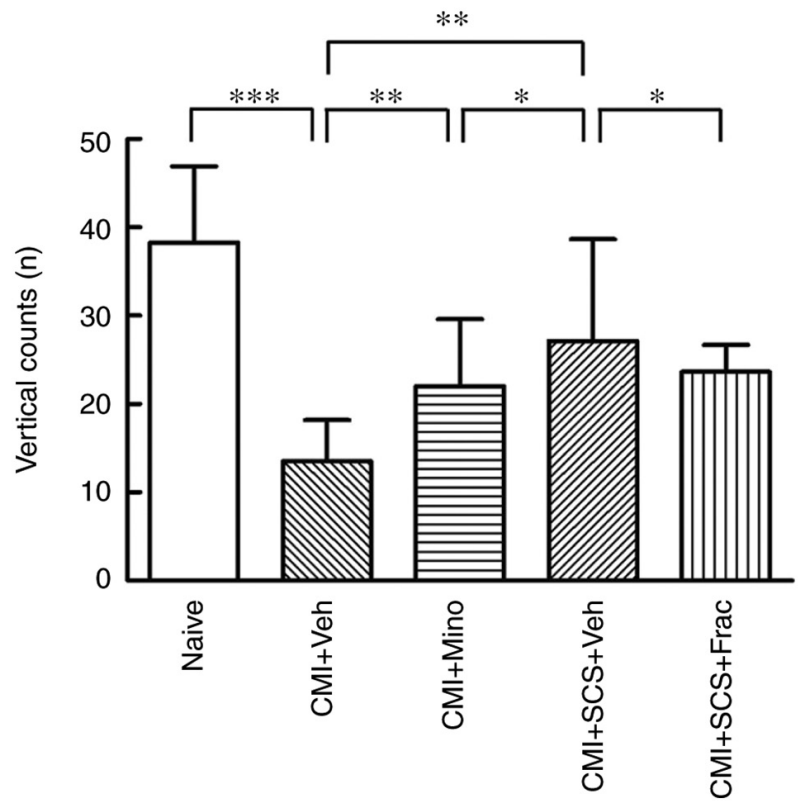

Figure 8. Behavioral pharmacological assessments of CMI rats in open field tests. Microglial inhibition reduces CMI-induced cardiac pain, whereas microglial activation partially reverses the therapeutic effects of SCS on CMI model rats, as revealed by the open field test. The declined vertical count in CMI model rats was increased by i.t. Mino administration. This therapeutic effect was weaker compared with that of SCS treatment on CMI model rats. However, following i.t. Frac administration, the vertical count of CMI model rats after $\mathrm{SCS}$ was decreased. $\mathrm{N}=8$ rats/group. ${ }^{*} \mathrm{P}<0.05,{ }^{* * *} \mathrm{P}<0.01,{ }^{* * *} \mathrm{P}<0.001$. CMI, chronic myocardial ischemia; Frac, fractalkine; i.t., intrathecal; Mino, minocycline; SCS, spinal cord stimulation; Veh, vehicle.

activation in a chronic constriction injury model (38). It was suggested that this discrepancy may partly be due to differences in animal models, post-surgery time points and SCS protocols among the studies.

How SCS inhibits spinal microglia and relieves cardiac pain remains unknown. Interestingly, other neuromodulation therapeutic treatments, including electroacupuncture (40) and transcutaneous electrical nerve stimulation (41), have been reported to reduce glial activation. It has been proven that SCS could increase the release of the inhibitory neurotransmitters $\gamma$-aminobutyric acid (GABA), serotonin, acetylcholine and opioids in the spinal dorsal horn (23). Microglia express corresponding inhibitory neurotransmitter receptors, including GABAergic, serotonergic and opioidergic receptors, and thus are directly deactivated by such release $(42,43)$. Alternatively, these inhibitory neurotransmitters could also reduce the release of neuronal excitatory neurotransmitters, such as glutamate and ATP, which in turn decrease glial cell activity indirectly (44). Specifically, it has been demonstrated that $\mathrm{P} 2 \mathrm{X}$ purinoceptor 7 ( $\mathrm{P} 2 \mathrm{X} 7)$ and $\mathrm{P} 2 \mathrm{X} 4$ are expressed on microglia $(45,46)$. Once these purinergic receptors are activated by ATP, the MAPK pathway (including p38) is phosphorylated, and the downstream pro-inflammatory mediators IL- $1 \beta$ and TNF- $\alpha$ are synthesized and influence neurons to perpetuate the nociceptive response. Conversely, SCS inhibits chronic cardiac pain partially by inhibiting microglial p38 MAPK and its downstream pro-inflammatory mediators, such as IL- $1 \beta$ and TNF- $\alpha$. Consequently, besides the well-known reinforcing 'gate-control' effect, we proposed that SCS could also modulate glial-neuronal interactions to reverse central sensitization in chronic cardiac pain conditions.

The present study has some limitations. As it only used conventional SCS, the effects of other SCS paradigms, such as high-frequency, sub-sensory threshold and burst SCS, on chronic cardiac pain require further investigation. Moreover, transgenic animals should be introduced to confirm the role of SCS in such a chronic cardiac pain model.

In summary, the present study provided evidence that SCS reduces chronic cardiac pain partially by inhibiting the spinal microglial p38 MAPK pathway and thus potentially downregulating the expression of pro-inflammatory mediators, such as IL- $1 \beta$ and TNF- $\alpha$. Therefore, SCS therapy may be a promising way to treat intractable chronic angina pectoris.

\section{Acknowledgements}

Not applicable.

\section{Funding}

This study was supported by The National Natural Science Foundation of China (grant no. 81701115) and The Key Research and Development Program of Shannxi Province (grant no. 2020SF-242).

\section{Availability of data and materials}

The datasets used and/or analyzed during the current study are available from the corresponding author on reasonable request.

\section{Authors' contributions}

JW, YQL and JBZ designed the study, wrote the manuscript and confirmed the authenticity of the raw data. JW, XCW and MMZ completed the animal experiments and the statistical analysis. JHR and YS participated in the morphological and behavioral experiments. JZL participated in the western blot analysis. XQW and SYH participated in statistical analysis. All authors read and approved the final manuscript.

\section{Ethics approval and consent to participate}

Animal experiments were performed in accordance with the ethical guidelines of the International Association for the Study of Pain and were approved by the Animal Use and Care Committee for Research and Education at the General Hospital of Western Command Theater and Air Force Military Medical University (Chengdu, China; approval no. 10070).

\section{Patient consent for publication}

Not applicable.

\section{Competing interests}

The authors declare that they have no competing interests. 


\section{References}

1. Waltenberger J: Chronic refractory angina pectoris: Recent progress and remaining challenges. Eur Heart J 38: 2556-2558, 2017.

2. Hale J, Bailey-Classen A and Cheng J: Spinal cord stimulation for refractory angina pectoris. Pain Med 21: 198-200, 2020 .

3. Foreman RD, Garrett KM and Blair RW: Mechanisms of cardiac pain. Compr Physiol 5: 929-960, 2015.

4. Rosen SD, Paulesu E, Wise RJ and Camici PG: Central neural contribution to the perception of chest pain in cardiac syndrome X. Heart 87: 513-519, 2002

5. Rosen SD: From heart to brain: The genesis and processing of cardiac pain. Can J Cardiol 28 (2 Suppl): S7-S19, 2012.

6. Li J, Zhang MM, Tu K, Wang J, Feng B, Zhang ZN, Lei J, Li YQ, Du JQ and Chen T: The excitatory synaptic transmission of the nucleus of solitary tract was potentiated by chronic myocardial infarction in rats. PLoS One 10 e0118827, 2015

7. Basbaum AI, Bautista DM, Scherrer G and Julius D: Cellular and molecular mechanisms of pain. Cell 139: 267-284, 2009.

8. Lanza GA, Grimaldi R, Greco S, Ghio S, Sarullo F, Zuin G De Luca A, Allegri M, Di Pede F, Castagno D, et al: Spinal cord stimulation for the treatment of refractory angina pectoris: A multicenter randomized single-blind study (the SCS-ITA trial). Pain 152: 45-52, 2011.

9. Pan X, Bao H, Si Y, Xu C, Chen H, Gao X, Xie X, Xu Y, Sun F and Zeng L: Spinal cord stimulation for refractory angina pectoris: A systematic review and meta-analysis. Clin J Pain 33: 543-551, 2017.

10. Marchand S: Spinal cord stimulation analgesia: Substantiating the mechanisms for neuropathic pain treatment. Pain 156: 364-365, 2015.

11. Sato KL, Johanek LM, Sanada LS and Sluka KA: Spinal cord stimulation reduces mechanical hyperalgesia and glial cell activation in animals with neuropathic pain. Anesth Analg 118: 464-472, 2014

12. Zimmermann M: Ethical guidelines for investigations of experimental pain in conscious animals. Pain 16: 109-110, 1983

13. Chen J, Zhan Y, Wang Y, Han D, Tao B, Luo Z, Ma S, Wang Q, Li X, Fan L, et al: Chitosan/silk fibroin modified nanofibrous patches with mesenchymal stem cells prevent heart remodeling post-myocardial infarction in rats. Acta Biomater 80: 154-168, 2018.

14. Zhang MM, Liu SB, Chen T, Koga K, Zhang T, Li YQ and Zhuo M: Effects of NB001 and gabapentin on irritable bowel syndrome-induced behavioral anxiety and spontaneous pain. Mol Brain 7: 47, 2014.

15. King T, Vera-Portocarrero L, Gutierrez T, Vanderah TW, Dussor G, Lai J, Fields HL and Porreca F: Unmasking the tonic-aversive state in neuropathic pain. Nat Neurosci 12: 1364-1366, 2009.

16. Wang J, Feng DY, Li ZH, Feng B, Zhang H, Zhang T, Chen T and Li YQ: Activation of the mammalian target of rapamycin in the rostral ventromedial medulla contributes to the maintenance of nerve injury-induced neuropathic pain in rat. Neural Plast 2015 : $394820,2015$.

17. Zhuang ZY, Wen YR, Zhang DR, Borsello T, Bonny C Strichartz GR, Decosterd I and Ji RR: A peptide c-Jun N-terminal kinase (JNK) inhibitor blocks mechanical allodynia after spinal nerve ligation: Respective roles of JNK activation in primary sensory neurons and spinal astrocytes for neuropathic pain development and maintenance. J Neurosci 26: 3551-3560, 2006.

18. Liao YH, Wang J, Wei YY, Zhang T, Zhang Y, Zuo ZF, Teng XY and Li YQ: Histone deacetylase 2 is involved in $\mu$-opioid receptor suppression in the spinal dorsal horn in a rat model of chronic pancreatitis pain. Mol Med Rep 17: 2803-2810, 2018.

19. Livak KJ and Schmittgen TD: Analysis of relative gene expression data using real-time quantitative PCR and the 2(-Delta Delta C(T)) Method. Methods 25: 402-408, 2001

20. Bhandare AM, Kapoor K, Powell KL, Braine E, Casillas-Espinosa P, O'Brien TJ, Farnham MMJ and Pilowsky PM: Inhibition of microglial activation with minocycline at the intrathecal level attenuates sympathoexcitatory and proarrhythmogenic changes in rats with chronic temporal lobe epilepsy. Neuroscience 350: 23-38, 2017.
21. Liu PY, Lu CL, Wang CC, Lee IH, Hsieh JC, Chen CC, Lee HF, Lin HC, Chang FY and Lee SD: Spinal microglia initiate and maintain hyperalgesia in a rat model of chronic pancreatitis. Gastroenterology 142: 165-173.e2, 2012.

22. Wang J, Li ZH, Feng B, Zhang T, Zhang H, Li H, Chen T, Cui J, Zang WD and Li YQ: Corticotrigeminal projections from the insular cortex to the trigeminal caudal subnucleus regulate orofacial pain after nerve injury via extracellular signal-regulated kinase activation in insular cortex neurons. Front Cell Neurosci 9: 493, 2015

23. Sdrulla AD, Guan Y and Raja SN: Spinal cord stimulation: Clinical efficacy and potential mechanisms. Pain Pract 18: 1048-1067, 2018

24. Dones I and Levi V: Spinal cord stimulation for neuropathic pain: Current trends and future applications. Brain Sci 8: 138, 2018.

25. Chen J, Winston JH, Fu Y, Guptarak J, Jensen KL, Shi XZ, Green TA and Sarna SK: Genesis of anxiety, depression, and ongoing abdominal discomfort in ulcerative colitis-like colon inflammation. Am J Physiol Regul Integr Comp Physiol 308: R18-R27, 2015.

26. Ji RR, Chamessian A and Zhang YQ: Pain regulation by non-neuronal cells and inflammation. Science 354: 572-577, 2016.

27. Berta T, Qadri YJ, Chen G and Ji RR: Microglial signaling in chronic pain with a special focus on caspase 6, p38 MAP kinase, and sex dependence. J Dent Res 95: 1124-1131, 2016.

28. Chen G, Zhang YQ, Qadri YJ, Serhan CN and Ji RR: Microglia in pain: Detrimental and protective roles in pathogenesis and resolution of pain. Neuron 100: 1292-1311, 2018.

29. Wang W, Mei XP, Chen L, Tang J, Li JL, Wu SX, Xu LX and Li YQ: Triptolide prevents and attenuates neuropathic pain via inhibiting central immune response. Pain Physician 15: E995-E1006, 2012.

30. Mai L, Zhu X, Huang F, He H and Fan W: p38 mitogen-activated protein kinase and pain. Life Sci 256: 117885, 2020.

31. Kiyomoto M, Shinoda M, Honda K, Nakaya Y, Dezawa K, Katagiri A, Kamakura S, Inoue T and Iwata K: p38 phosphorylation in medullary microglia mediates ectopic orofacial inflammatory pain in rats. Mol Pain 11: 48, 2015

32. Taves S, Berta T, Liu DL, Gan S, Chen G, Kim YH, Van de Ven T, Laufer S and Ji RR: Spinal inhibition of p38 MAP kinase reduces inflammatory and neuropathic pain in male but not female mice: Sex-dependent microglial signaling in the spinal cord. Brain Behav Immun 55: 70-81, 2016.

33. Castany S, Gris G, Vela JM, Verdu E and Boadas-Vaello P: Critical role of sigma-1 receptors in central neuropathic pain-related behaviours after mild spinal cord injury in mice. Sci Rep 8: 3873, 2018

34. Park KE and Conti CR: Non-PCI/CABG therapies for refractory angina. Trends Cardiovasc Med 28: 223-228, 2018.

35. Saraste A, Ukkonen H, Varis A, Vasankari T, Tunturi S, Taittonen M, Rautakorpi P, Luotolahti M, Airaksinen KE and Knuuti J: Effect of spinal cord stimulation on myocardial perfusion reserve in patients with refractory angina pectoris. Eur Heart J Cardiovasc Imaging 16: 449-455, 2015

36. Lopshire JC and Zipes DP: Spinal cord stimulation for heart failure: Preclinical studies to determine optimal stimulation parameters for clinical efficacy. J Cardiovasc Transl Res 7: 321-329, 2014.

37. Vallejo R, Kelley CA, Gupta A, Smith WJ, Vallejo A and Cedeno DL: Modulation of neuroglial interactions using differential target multiplexed spinal cord stimulation in an animal model of neuropathic pain. Mol Pain 16: 1744806920918057, 2020.

38. Shu B, He SQ and Guan Y: Spinal cord stimulation enhances microglial activation in the spinal cord of nerve-injured rats. Neurosci Bull 36: 1441-1453, 2020.

39. Dong X, Li H, Lu J, Yang Y, Jing H, Cheng Y, Jin M and Cheng W: Spinal cord stimulation postconditioning reduces microglial activation through down-regulation of ERK1/2 phosphorylation during spinal cord ischemic reperfusion in rabbits. Neuroreport 29: 1180-1187, 2018.

40. Liang Y, Qiu Y, Du J, Liu J, Fang J, Zhu J and Fang J: Inhibition of spinal microglia and astrocytes contributes to the anti-allodynic effect of electroacupuncture in neuropathic pain induced by spinal nerve ligation. Acupunct Med 34: 40-47, 2016.

41. Matsuo H, Uchida K, Nakajima H, Guerrero AR, Watanabe S, Takeura N, Sugita D, Shimada S, Nakatsuka T and Baba H: Early transcutaneous electrical nerve stimulation reduces hyperalgesia and decreases activation of spinal glial cells in mice with neuropathic pain. Pain 155: 1888-1901, 2014. 
42. Maduna T, Audouard E, Dembele D, Mouzaoui N, Reiss D, Massotte D and Gaveriaux-Ruff C: Microglia express Mu opioid receptor: Insights from transcriptomics and fluorescent reporter mice. Front Psychiatry 9: 726, 2019.

43. El Oussini H, Bayer H, Scekic-Zahirovic J, Vercruysse P, Sinniger J, Dirrig-Grosch S, Dieterlé S, Echaniz-Laguna A, Larmet Y, Müller K, et al: Serotonin 2B receptor slows disease progression and prevents degeneration of spinal cord mononuclear phagocytes in amyotrophic lateral sclerosis. Acta Neuropathol 131: 465-480, 2016.

44. Chen T, Willoughby KA and Ellis EF: Group I metabotropic receptor antagonism blocks depletion of calcium stores and reduces potentiated capacitative calcium entry in strain-injured neurons and astrocytes. J Neurotrauma 21: 271-281, 2004.
45. Trang T, Beggs S, Wan X and Salter MW: P2X4receptor-mediated synthesis and release of brain-derived neurotrophic factor in microglia is dependent on calcium and p38-mitogen-activated protein kinase activation. J Neurosci 29: 3518-3528, 2009.

46. Fan X, Ma W, Zhang Y and Zhang L: P2X7 receptor (P2X7R) of microglia mediates neuroinflammation by regulating (NOD)-like receptor protein 3 (NLRP3) inflammasome-dependent inflammation after spinal cord injury. Med Sci Monit 26: e925491, 2020.

This work is licensed under a Creative Commons Attribution-NonCommercial-NoDerivatives 4.0 International (CC BY-NC-ND 4.0) License. 